\title{
Bilateral Luxatio Erecta: A Case Report
}

\author{
Jason Lippert, Bobby Desai \\ Ocala Regional Emergency Department, Ocala Regional Medical Center, Ocala, USA \\ Email: ocalaemergencymedicine@gmail.com
}

How to cite this paper: Lippert, J. and Desai, B. (2018) Bilateral Luxatio Erecta: A Case Report. Case Reports in Clinical Medicine, 7, 608-612.

https://doi.org/10.4236/crcm.2018.712056

Received: October 4, 2018

Accepted: December 15, 2018

Published: December 18, 2018

Copyright $\odot 2018$ by authors and Scientific Research Publishing Inc.

This work is licensed under the Creative

Commons Attribution International

License (CC BY 4.0).

http://creativecommons.org/licenses/by/4.0/

\begin{abstract}
Luxatio erecta is a highly uncommon type of shoulder dislocation, representing approximately $0.5 \%$ of all shoulder dislocations fitting into this category. In this paper, we present the case of a patient who presented post trauma with bilateral inferior glenohumeral joint dislocations (also called Luxatio Erecta). Considering that Luxatio Erecta only has a $0.5 \%$ chance of being present with a unilateral dislocation, the probability of a patient presenting 2 of these dislocations at the same time is approximately $0.25 \%(0.5 \% \times 0.5 \%=0.25 \%)$. In addition to this, Luxatio Erecta frequently presents with injuries to the brachial plexus and/or a humeral fracture. Despite this, neither of our patient's dislocations were associated with any fractures or neurovascular injury and both were successfully reduced the Emergency Department. Both the patient's presentation and outcome are quite uncommon, which makes this case an invaluable opportunity to go over the unique characteristics to Luxatio Erecta.
\end{abstract}

\section{Keywords}

Luxatio Erecta, Closed Reduction

\section{Introduction}

Luxatio Erecta is a rare type of shoulder dislocation, accounting for approximately $0.5 \%$ of all shoulder dislocations [1]. It is associated with traumatic injuries that hyperabduct the arm, which forces the proximal humerus into the acromion, which allows the humeral head to disengage from the glenoid.

Classically, physical exam will show the affected arm hyperabducted with flexion at the elbow, and hand positioning superior or posterior to the patient's head. The examiner should be able to palpate the humeral head in the patient's axilla, along with an empty glenoid cavity [1].

\section{Case Presentation}

A 70 year old male presented to our Emergency Department (ED) post traumat- 
ic fall. Patient was hanging outdoor Christmas lights, when his ladder fell out from under him. Patient held onto the edge of his roof, hanging from his arms before he fell and landed on his back. Patient denied neck/back pain, along with loss of consciousness. Was given $10 \mathrm{mg}$ of Morphine by EMS prior to ED presentation?

Presenting vitals in the ED were pulse of $68 \mathrm{bpm}$, respiratory rate of 18 , and blood pressure of $158 / 74 \mathrm{mmHg}$. On physical exam, the patient had bilateral abducted and flexed arms, with his $\mathrm{L}$ hand superior to his head and his $\mathrm{R}$ hand superior + posterior to his head; empty glenoid cavity bilaterally, palpable humeral head in the $L$ axilla. No significant neurological or vascular deficiencies were found for both extremities. No other significant findings were present on exam. (Table 1)

Bilateral shoulder and humeral head $\mathrm{x}$-rays were ordered. Shoulder $\mathrm{x}$-rays demonstrated bilateral, inferior dislocations of the humeral head (Luxatio Erecta), no fractures were identified on the shoulder or humeral x-rays (Figure 1 and Figure 2). However, due to the patient's body habitus, and his bilateral dislocations, proper positioning could not be obtained for the R humeral $\mathrm{x}$-ray, and a fracture could not be entirely ruled out.

Prior to closed reduction of the both glenohumeral joints, we had a conversation with patient and his spouse concerning the risk for additional injury should there be an unseen fracture of his right humeral head. The patient and his spouse understood, and consented to closed reduction of both shoulders.

Closed reduction was carried out under Conscious Sedation with Ketamine, using Traction-Counter-traction technique. A sheet was wrapped around the $\mathrm{L}$ shoulder and both ends were pulled together at the $\mathrm{R}$ hip. The $1^{\text {st }}$ physician was positioned at the head of the bed holding the proximal $\mathrm{L}$ arm, while the $2^{\text {nd }}$ was positioned the $\mathrm{R}$ waist, holding both ends of the sheet. The $1^{\text {st }}$ physician pulled on L proximal arm, creating axial traction, while the $2^{\text {nd }}$ physician pulled on the sheet, creating counter-traction. During this, the $1^{\text {st }}$ physician increased the intensity of abduction in the affected limb to provide additional pressure on the humeral head. L shoulder was successfully reduced, and the R shoulder was successfully reduced with a setup mirrored to this one. Neurovascular function remained intact bilaterally post reduction, and post reduction x-rays demonstrated no fractures of humeral head (Figure 1 and Figure 2). Patient was placed in bilateral slings and discharged home with 1 week follow up with Orthopedic Surgery.

\section{Discussion}

Luxatio Erecta is a truly uncommon type of shoulder dislocation and it is important for an Emergency Physician to be aware of its presence when seen. A physician should know how to properly reduce Luxatio Erecta, as most instances can be successfully treated with non-operative management, but also understand when surgical care is required. In addition, it is vital to remember the associated risks and complications associated with this injury. 
Table 1. Summarizes clinical characteristics of patient presentation.

HPI: 70 year old male s/p traumatic fall from a ladder, landing on his back. No LOC, back or neck pain. $10 \mathrm{mg}$ IV Morphine from EMS.

Vitals: 68 BPM, RR 18, BP 158/74 mmHG.

Physical exam: Bilateral abducted and flexed arms, $\mathrm{L}$ hand superior to head and $\mathrm{R}$ hand is superior + posterior to head. BL empty glenoid cavity. Neurovascular function intact bilaterally.

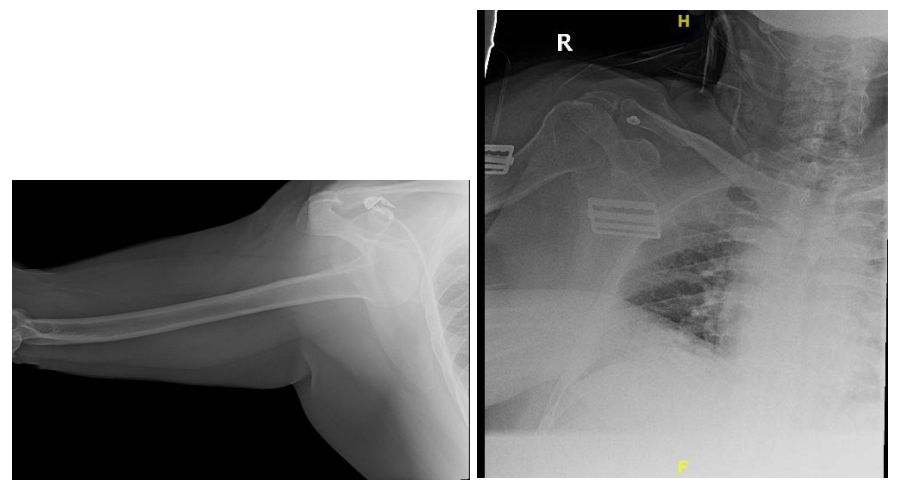

Figure 1. Left image: Right sided Luxatio Erecta, pre-reduction Right image: Right sided Luxatio Erecta, post-reduction using Traction-Counter-traction technique.

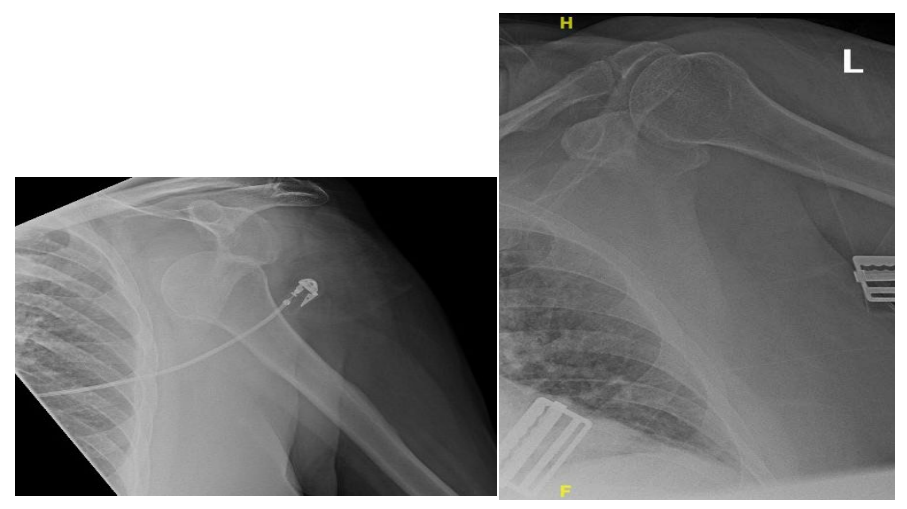

Figure 2. Left image: Left sided Luxatio Erecta, pre-reduction Right image: Left sided Luxatio Erecta, post-reduction using Traction-Counter-traction technique.

\subsection{Typical Presentation}

On presentation, Luxatio Erecta will have the affected extremity held above or behind the patient's head, with the elbow flexed and abducted. The glenoid cavity is empty and the physician may palpate the humeral head in the axilla. On $\mathrm{x}$-ray, the humeral head will be inferior to the rim of the glenoid and the humeral shaft is parallel to the scapular spine [2].

\subsection{Reduction Techniques}

At the time of writing, there are two methods for closed reduction of Luxatio Erecta; Traction-Counter-traction and The Two-Step Maneuver. 


\section{Traction-Counter-traction:}

With an assistant, wrap a sheet around the affected shoulder, with both ends of the sheet pulled towards the contralateral hip. This provides the counter-traction required. Following this, straighten the affected elbow while keeping the arm fully abducted. Then pull in line with humeral shaft (if required, the assistant may apply additional force to the humeral head in a cephalad and lateral direction). When the humeral head is reduced into glenoid fossa, slowly adduct the shoulder towards the body.

The Two-Step Maneuver:

Inferior Dislocation $\rightarrow$ Anterior Dislocation $\rightarrow$ Reduction.

On the affected side, push on the lateral aspect of the midshaft humerus, and pull on the medial epicondyle of the elbow. This converts the inferior dislocation to an anterior dislocation. Once this has been accomplished, the physician may reduce the anterior dislocation with a number of maneuvers [3] (two examples being the Milch technique or Scapular manipulation).

\subsection{Reduction Tips}

Always ensure adequate relaxation and sedation prior to reduction attempt.

Just like other closed reductions, a thorough pre and post reduction neurological exam is essential.

Always obtain post reduction $\mathrm{x}$-rays.

\subsection{Complications}

With an inferior dislocation, there is an associated risk of rotator cuff tear, with some citing a $12 \%$ incidence with Luxatio Erecta [4] [5]. Luxatio Erecta can also present with concomitant fracture of the greater tuberosity. An article from the Journal of Orthopedic trauma reviewed 80 cases of Luxatio Erecta and found that $80 \%$ presented with a rotator cuff tear or a fracture of the greater tuberosity [5]. While our patient had no neurovascular abnormalities, the same article estimates that $50 \%-60 \%$ of Luxatio Erecta patients have an associated Brachial Plexus Injury [5]. Considering this, a thorough and well documented neurological exam of the affected extremity should be performed before and after reduction. The same can be said for a vascular exam, due to risk of injury to the axillary artery [6].

\subsection{Surgical Intervention}

Despite the high rate of success, Luxatio Erecta cannot be universally treated with closed reduction. Should the dislocation by irreducible, open or associated with vascular injury, the patient will require full surgical intervention. In addition, a fracture of the acromion, clavicle, inferior glenoid fossa and greater tuberosity will require surgical management [7].

\section{Conflicts of Interest}

The authors declare no conflicts of interest. 


\section{References}

[1] Imerci, A., Gölcük, Y., Ugur, S.G., Ursavas, H.T., Savran, A. and Sürer, L. (2013) Inferior Glenohumeral Dislocation (Luxatio Erecta Humeri): Report of Six Cases and Review of the Literature. Ulus Travma Acil Cerrahi Derg, 19, 41-44. https://doi.org/10.5505/tjtes.2013.35305

[2] Kothari, K., Berstein, R.M., Griffiths, H.J., et al. (1984) Luxatio Erecta. Skeletal Radiology, 11, 47-49. https://doi.org/10.1007/BF00361132

[3] Nho, S.J., Dodson, C. and Bardzik, K.F. (2006) The Two-Step Maneuver for Closed Reduction of Inferior Glenohumeral Dislocation (Luxatio Erecta to Anterior Dislocation to Reduction). JOT, 20, 354-357

[4] Grate Jr., I. (2000) Luxatio Erecta: A Rarely Seen, but Often Missed Shoulder Dislocation. AJEM, 18, 317-321. https://doi.org/10.1016/S0735-6757(00)90127-X

[5] Mallon, W.J., Bassett 3rd, F.H. and Goldner, R.D. (1990) Luxatio Erecta: The Inferior Glenohumeral Dislocation. Journal of Orthopaedic Trauma, 4, 19-24. https://doi.org/10.1097/00005131-199003000-00003

[6] Lev-El, A., Adar, R. and Rubinstein, Z. (1981) Axillary Artery Injury in Erect Dislocation of the Shoulder. The Journal of Trauma, 21, 323-325. https://doi.org/10.1097/00005373-198104000-00011

[7] Freundlich, B.D. (1983) Luxatio Erecta. The Journal of Trauma, 23, 434-436. https://doi.org/10.1097/00005373-198305000-00015 